\title{
Numerical counterexamples of Lorenz system in implicit time scheme
}

\author{
X. Chen \\ Numerical Analysis Research Center, GZ 510510, China.
}

\begin{abstract}
In nonlinear self-consistent system, Lorenz system (Lorenz equations) is a classic case with chaos solutions which are sensitively dependent on the initial conditions. As it is difficult to get the analytical solution, the numerical methods and qualitative analytical methods are widely used in many studies. In these papers, Runge-Kutta method is the one most often used to solve these differential equations. However, this method is still a method based on explicit time scheme, which would be the main reason for the chaotic solutions to Lorenz system. In this work, numerical experiments based on implicit time scheme and explicit scheme are setup for comparison, the results show that: in implicit time scheme, the numerical solutions (counterexamples) are without chaos; for an original volume, the volume shrinks exponentially fast to 0 in common.
\end{abstract}

Key words: Lorenz system; implicit time scheme; counterexamples

\section{Introduction}

Chaos is one of the most important scientific achievements, and is also very important in modern nonlinear science. Lorenz [1] got a much simpler and easier-to-analyze system (Lorenz system). Later, numerical solutions of the Lorenz system and behavior of Lorenz flow are analyzed in many studies $[2,3,4,5,6,7,8]$; for computing the numerical methods, the explicit time schemes are widely used; and in numerical methods, Runge-Kutta method is the widely used. For the numerical solutions of Lorenz system, the solutions sensitively dependent on the initial conditions are a very important phenomenon, which has an effect on numerical weather prediction $[9,10]$. But most of these numerical solutions are computed on the basis of explicit time schemes and the very less are based on implicit time schemes.

This paper is organized as follows. In Section 2, we will present a brief introduction to Lorenz system and numerical scheme applied in this paper. The numerical results are shown in Section 3. Discussion and conclusions

* Corresponding author: Xuan Chen (E-mail: chzffx@qq.com or chenxuan@mail.iap.ac.cn). 
are presented in Section 4.

\section{Introduction to Lorenz System and numerical scheme}

The Lorenz equations are

$$
\left\{\begin{array}{l}
\dot{X}=-\sigma(X-Y) \\
\dot{Y}=\rho X-Y-X Z, \\
\dot{Z}=X Y-\beta Z
\end{array}\right.
$$

where the parameters $\sigma, \rho$, and $\beta$ are kept constant within integration. The particular parameter values chosen by Lorenz [1]: $\sigma=10, \rho=28$, and $\beta=8 / 3$, which result in chaotic solutions. Although Lorenz system is a much simpler and easier-to-analyze system, it is very hard to get the analytical solutions as with two nonlinear terms. However, Gilberto et al. [11] applied an analytical approach to solve the Lorenz system without chaos. The explicit solution maybe wrong as the solution is also chaotic with the initial conditions $(-6 \sqrt{2}+\varepsilon,-6 \sqrt{2}+\varepsilon, 27+\varepsilon)$ as $\sigma$ $=10, \rho=28, \beta=8 / 3$, and $\varepsilon=O\left(10^{-20}\right)$.

On the basis of the qualitative analytical methods and numerical methods, much progress has been made in the study of Lorenz system. With the qualitative analytical methods, a lot of analytical scheme or exponents are applied in the geometric morphology analysis (the convergence domains, attractors, et al.), such as Lyapunov exponents, limit cycle, perturbation theory and so on. In numerical methods, Runge-Kutta method is widely used to solve Lorenz system.

Taking the Runge-Kutta 4th order method as an example, for an integrand $F(\dot{F}=G(F))$, on the previous point Ft, the next point $F_{t+1}$ can be written as:

$$
F_{t+1}=F_{t}+\Delta\left(\frac{k_{t}^{1}+2 k_{t}^{2}+2 k_{t}^{3}+k_{t}^{4}}{6}\right)
$$

where $\Delta$ is time step, and $k_{t}^{i}(i=1,2,3,4)$ are

$$
\left\{\begin{array}{l}
k_{t}^{1}=G\left(t, F_{t}\right) \\
k_{t}^{2}=G\left(t+\frac{\Delta}{2}, F_{t}+\frac{\Delta k_{t}^{1}}{2}\right) \\
k_{t}^{3}=G\left(t+\frac{\Delta}{2}, F_{t}+\frac{\Delta k_{t}^{2}}{2}\right) \\
k_{t}^{4}=G\left(t+\Delta, F_{t}+\Delta k_{t}^{3}\right)
\end{array} .\right.
$$

In implicit time scheme, the iterative equations for Lorenz system can be written as 


$$
\left\{\begin{aligned}
(1+\sigma \Delta) X_{t+1}-\sigma \Delta Y_{t+1}-X_{t} & =0 \\
(1+\Delta) Y_{t+1}-\rho \Delta X_{t+1}+\Delta X_{t+1} Z_{t+1}-Y_{t} & =0 \\
(1+\beta \Delta) Z_{t+1}-\Delta X_{t+1} Y_{t+1}-Z_{t} & =0
\end{aligned}\right.
$$

In numerical experiments in this work, $\Delta=0.01 \mathrm{~s}$ (too small time step may make the implicit solution transform to the explicit solution, the proof can be found in appendix); and simulation time is 1000s. In implicit time scheme, equations (1) can be solved by using the Optimization Theory [12].

\section{Numerical results}

In this section, the Runge-Kutta $4-5^{\text {th }}$ order method and implicit time scheme are applied in the three groups of contrastive experiments. The three parameters' values are chosen by Lorenz [1], $\sigma=10, \rho=28$, and $\beta=8 / 3$. In Table 1 we present initial conditions for different groups of contrastive experiments. The phase figures are shown in Fig. 1, in explicit time scheme, these trajectories tend to circle around the stable equilibrium points but do not ever repeat the same curve twice.
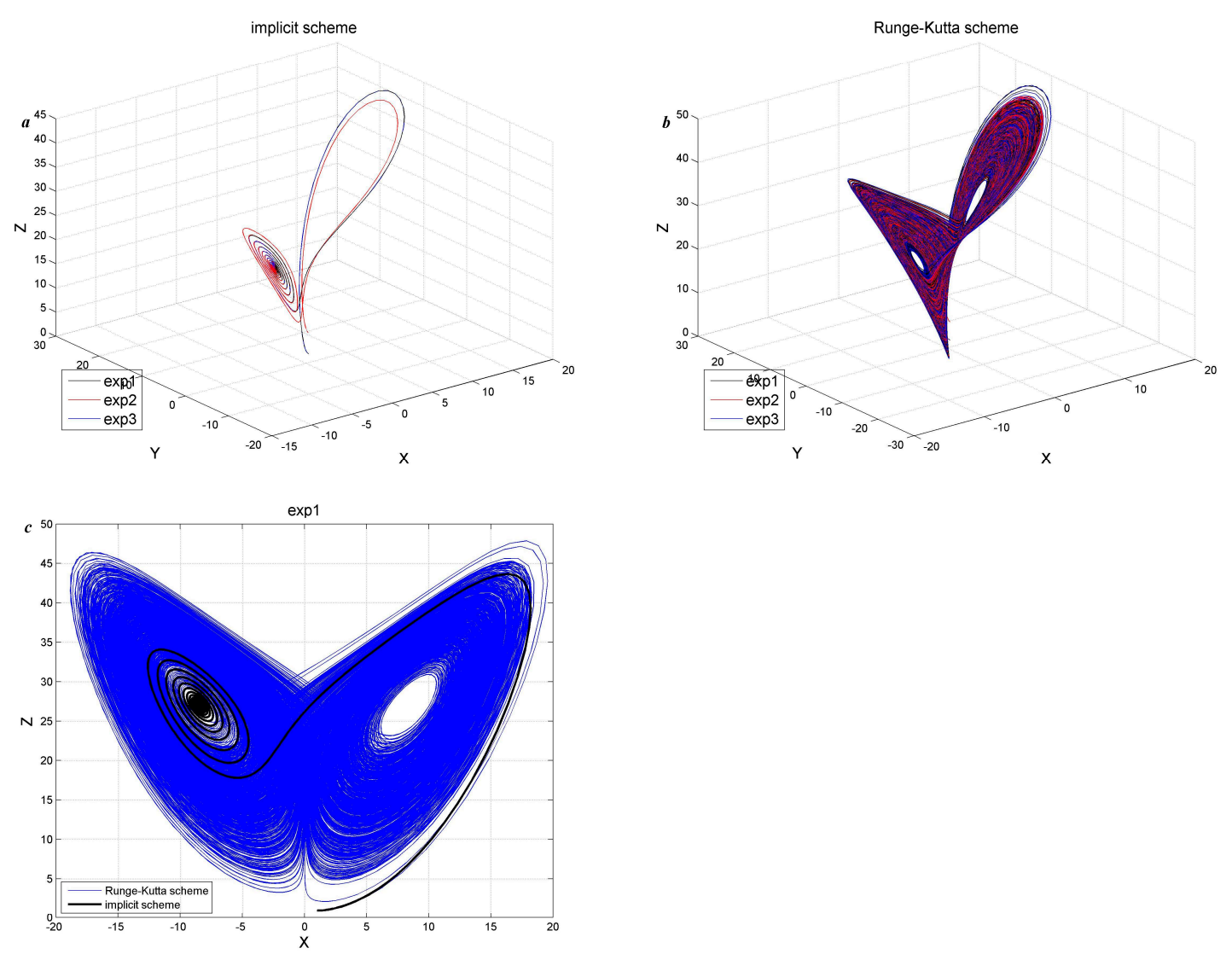

Fig. 1. Numerical solutions of the Lorenz equations, in (a) and (b), the black line with initial conditions $(1.0,1.0,1.0)$; the red line with initial conditions $(2.0,3.0,4.0)$; the green line with initial conditions $(1.0,1.3,1.0)$ : (a) Implicit solutions; (b) explicit solutions 
(the Runge-Kutta 4-5 $5^{\text {th }}$ order method). (c) Numerical solutions of the first group of contrastive experiment (the black line in implicit time scheme, the blue line in explicit time scheme.

Table 1. Initial conditions for different groups of contrastive experiments

\begin{tabular}{cccc}
\hline & $X(0)$ & $Y(0)$ & $Z(0)$ \\
\hline $\exp 1$ & 1.0 & 1.0 & 1.0 \\
$\exp 2$ & 2.0 & 3.0 & 4.0 \\
$\exp 3$ & 1.0 & 1.3 & 1.0 \\
\hline
\end{tabular}

Figure 1 shows that:

(1) In implicit time scheme, the numerical solution is not sensitive dependence on initial conditions, one of the main features of a chaotic system;

(2) In implicit time scheme, the trajectory converges to the value $(-8.4855,-8.4855,27.0004)$, which is the approximation to $(-6 \sqrt{2},-6 \sqrt{2}, 27)$; there are obvious shock exists in the phase Figure by the Runge-Kutta method (the explicit time scheme). It is also worth to mention that there are three equilibrium points in Lorenz system for the classical parameter values: $(0,0,0),(-6 \sqrt{2},-6 \sqrt{2}, 27)$ and $(6 \sqrt{2}, 6 \sqrt{2}, 27)$.

In addition, the change curves of each variables $(X, Y$ and $Z)$ are also compared. Here, the change curves of $X$ - $t$ are shown in Fig. 2.

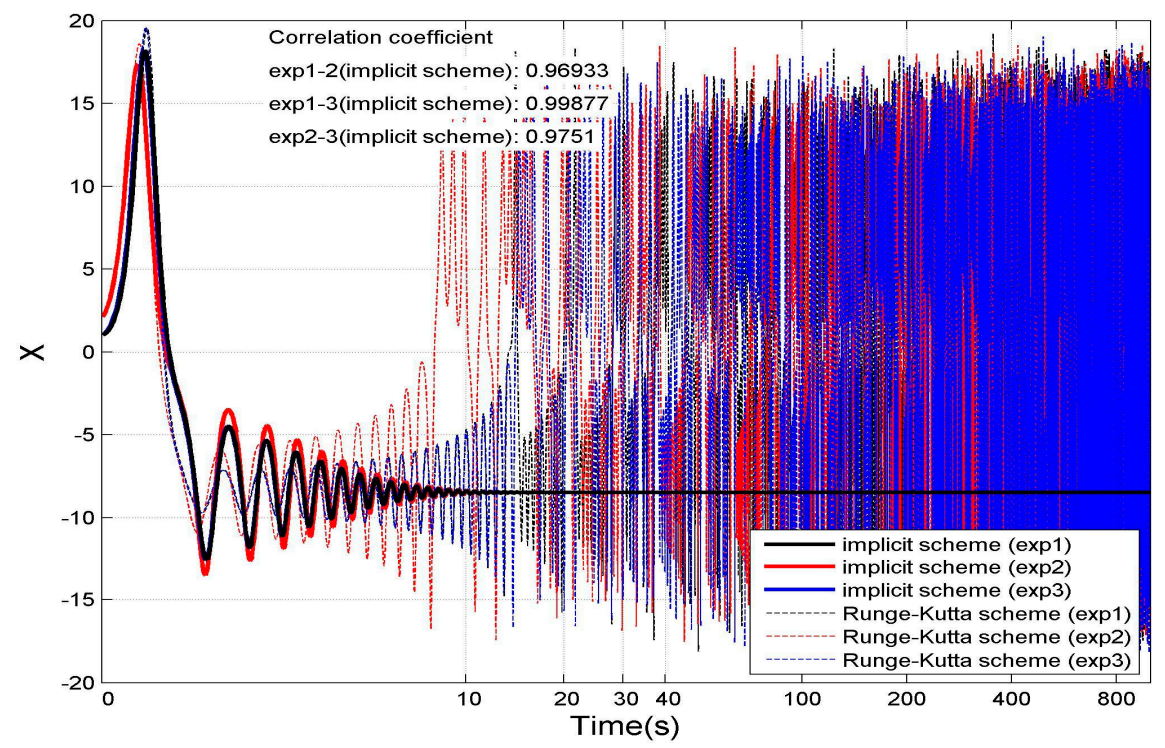

Fig. 2. Change curve of $X-t$ 
The change curves of $X-t$ (both two other are similar to the change curve of $X-t$ ) shows that: in the first few seconds, calculating results in both explicit time scheme (the Runge-Kutta $4-5^{\text {th }}$ order method) and implicit time scheme are similar in all experiments. Later, in implicit time scheme, the value of $X$ converges to -8.485 ; but, there are obvious shock exists in the change curve of $X$ - $t$ in explicit time scheme. We can also find that all the change curves of $X$ - $t$ in implicit time scheme are similar. On the basis of Fig. 2, we can conclude that:

(3) In implicit time scheme, the sensitivity dependence on initial conditions is non-existent.

Here, we defined the error expression representing calculating accuracy of single step calculation as below, in implicit time scheme

$$
\left\{\begin{array}{l}
\operatorname{err}_{X}=(1+\sigma \Delta) X_{t+1}-\sigma \Delta Y_{t+1}-X_{t} \\
\operatorname{err}_{Y}=(1+\Delta) Y_{t+1}-\rho \Delta X_{t+1}+\Delta X_{t+1} Z_{t+1}-Y_{t} \\
\operatorname{err}_{Z}=(1+\beta \Delta) Z_{t+1}-\Delta X_{t+1} Y_{t+1}-Z_{t}
\end{array}\right.
$$

and in explicit time scheme

$$
\left\{\begin{array}{l}
\operatorname{err}_{X}=X_{t+1}+(\sigma \Delta-1) X_{t}-\sigma \Delta Y_{t} \\
\operatorname{err}_{Y}=Y_{t+1}+(\Delta-1) Y_{t}-\rho \Delta X_{t}+\Delta X_{t} Z_{t} \\
\operatorname{err}_{Z}=Z_{t+1}+(\beta \Delta-1) Z_{t}-\Delta X_{t} Y_{t}
\end{array}\right.
$$

As Runge-Kutta scheme is also an iterative method in single step calculation, the error expression is only for qualitative reference only, which means that the error is not the real calculating error. But the error expressions in implicit time scheme are the calculating errors in single step calculation. Both accuracies in each step calculation with initial conditions $(1.0,1.0,1.0)$ are shown in Fig. 3, and the others are similar to Fig. 3.
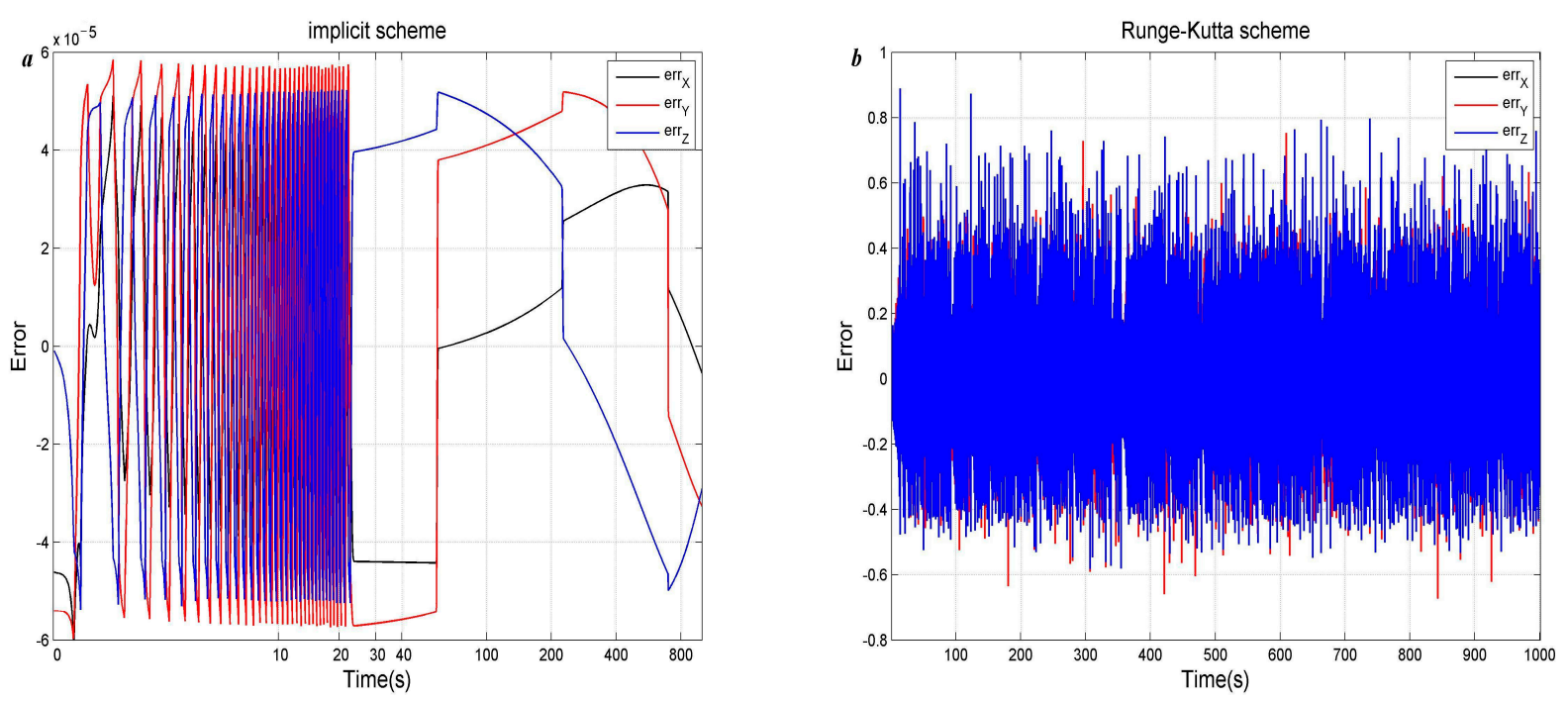

Fig. 3. Accuracies in each step calculation with initial conditions (1.0, 1.0, 1.0). 
Figure 3 shows that: in implicit time scheme, in the first about 20 seconds, the error is very complex, but simple in the later. But there is no special period in the integral process in explicit time scheme. In addition, we can find that the absolute errors are below $O\left(10^{-4}\right)$ in integral process (left part of Fig. 3). By Lax-Richtmyer Theorem, on the basis of Fig. 3, Eq. (1) can be seen as the approximation to Lorenz system. With analyzing the structure of Lorenz system, we can find that: no matter what discrete scheme applied in numerical analysis to Lorenz system, the numerical results do not contain points in the $Z$-axis.

There are three equilibrium points, all of implicit solutions converge to $(-6 \sqrt{2},-6 \sqrt{2}, 27)$. But both equilibrium points $(-6 \sqrt{2},-6 \sqrt{2}, 27)$ and $(6 \sqrt{2}, 6 \sqrt{2}, 27)$ are symmetrical and similar. So, why all of these numerical solutions converge to the equilibrium point $(-6 \sqrt{2},-6 \sqrt{2}, 27)$ is a very interesting problem. For this problem, we set up two implicit experiments with initial conditions $(100,178,-311)$ and $(-100,178,-311)$; the numerical solutions of each experiments converge to $(-6 \sqrt{2},-6 \sqrt{2}, 27)$ and $(6 \sqrt{2}, 6 \sqrt{2}, 27)$ respectively. On the basis of these, we indicate that there must be a symmetrical interface (the interface is not a plane, and we take a special mapping relation of $X$ and $Y$ as this interface), points (initial conditions) on both sides of which with slightly difference will converge to different equilibrium points. Compared to explicit time scheme (such as Runge-Kutta method), in implicit time scheme, the sensitivity dependence on initial conditions is non-existent may except points near the interface.

In Lorenz system, the divergence of the flow

$$
\frac{\partial \dot{X}}{\partial X}+\frac{\partial \dot{Y}}{\partial Y}+\frac{\partial \dot{Z}}{\partial Z}=-(\sigma+\beta+1)
$$

We define a volume matrix function $F v\left(t ; X_{i t}\right)(i=0,1,2,3)$

$$
F v\left(t ; X_{i t}\right)=\left[\begin{array}{ccc}
X_{1 t} & X_{2 t} & X_{3 t} \\
Y_{1 t} & Y_{2 t} & Y_{3 t} \\
Z_{1 t} & Z_{2 t} & Z_{3 t}
\end{array}\right]-\left[\begin{array}{ccc}
X_{0 t} & X_{0 t} & X_{0 t} \\
Y_{0 t} & Y_{0 t} & Y_{0 t} \\
Z_{0 t} & Z_{0 t} & Z_{0 t}
\end{array}\right]
$$

Here $i$ represents different experiments (expi) with different initial conditions, and $i=0$ means the experiment with initial conditions $(2.0,2.0,2.0)$. The Liouville's Theorem asserts that

$$
\dot{V}=\int_{D(t)} \frac{\partial \dot{X}}{\partial X}+\frac{\partial \dot{Y}}{\partial Y}+\frac{\partial \dot{Z}}{\partial Z} \mathrm{~d} X \mathrm{~d} Y \mathrm{~d} Z
$$

The volume $V(t)$ of a solid at time $t$ can be expressed [13] as 


$$
V(t)=V(0) \mathrm{e}^{-(\sigma+\beta+1) t}=\frac{1}{6}|\operatorname{det}(F v)| .
$$

Here, $\sigma+\beta+1 \approx 13.67$. So, the volume converges to the zeros as time increasing, which means most trajectories will converge to some special points when $\sigma+\beta+1>0$. If the numerical solutions with different initial conditions hold chaotic characteristics, the initial sensitivity may not make $V(t)$ converge to zero. Contrast to explicit solution, in implicit time scheme, the volume converges to zeros in the experiment (Fig. 4). By analyzing equation (2), with $\sigma+\beta+1>0$, we can find that Lorenz system is a dissipative system. In Fig. 4, we can indicate that the volume function $V(t)$ in implicit time scheme converges to zero in a very short time about 2 seconds. So, the chaotic solution may be caused by the numerical scheme in explicit time scheme. In addition, different trajectories cannot have common intersections, which include equilibrium points, such as the point $(-6 \sqrt{2},-6 \sqrt{2}, 27)$ in this work.
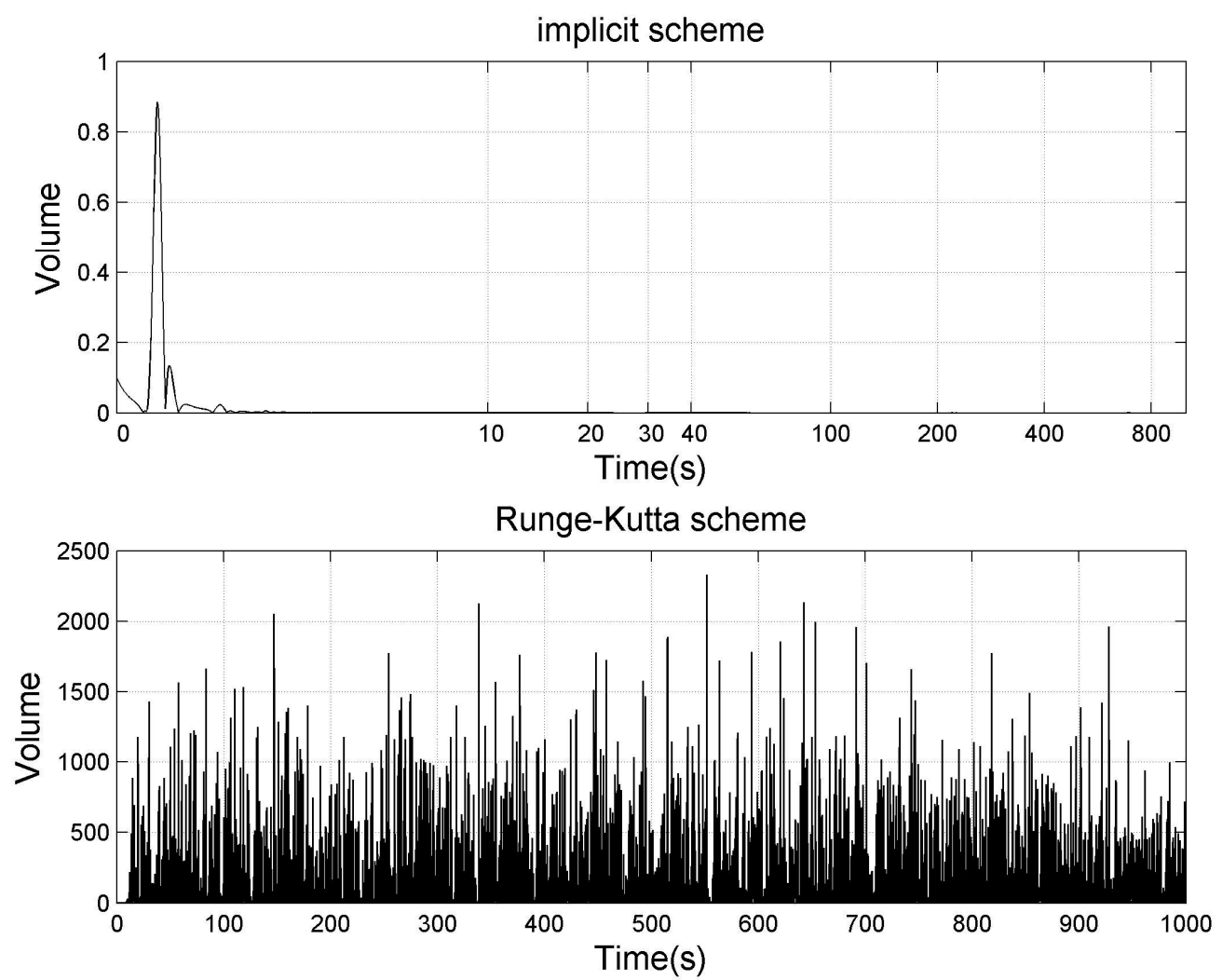

Fig. 4. Volumes series. The volumes are calculated by equation (2) with the points on the trajectories at the same time in different experiments with different initial values: $(1.0,1.0,1.0),(2.0,3.0,4.0),(1.0,1.3,1.0),(2.0,2.0,2.0)$.

\section{Discussion and conclusions}

In this work, on the basis of two numerical schemes, implicit scheme and explicit scheme (the Runge-Kutta $4-5^{\text {th }}$ order method), with different initial conditions, we find that there are many numerical counterexamples in 
implicit results. Numerical results show that: different types of numerical methods have an important influence on numerical solutions of Lorenz system. In implicit time scheme, there is no chaotic solution; compared to explicit scheme, there are less chaotic characteristics in the phase figures. On the basis of these, we can conclude that different types of numerical methods have a very important effect on the nonlinear equations' numerical solutions.

\section{Reference}

[1] E. N. Lorenz. Deterministic nonperiodic flow. Journal of the Atmospheric Sciences. 20, (1963), 130-141.

[2] D. A. Kaloshin. Search for and Stabilization of Unstable Saddle Cycles in the Lorenz System, Differential Equations. 11, (2001), 1636-1639.

[3] J. Biazar \& Z. Ayati. An Approximation to the Solution of the Brusselator System by Adomian Decomposition Method and Comparing the Results with Runge-Kutta Method. International Journal of contemporary Mathematical Sciences. 2, (2007), 983 - 989.

[4] N. Razali \& Ahmad R. Rozita. New Fifth-Order Runge-Kutta Methods for Solving Ordinary Differential Equation. Proceeding of Seminar on Engineering Mathematics. 2, (2008), 155-162.

[5] N. Razali \& Ahmad R. Rozita. Ahmad R. Solving Lorenz system by using Runge-Kutta method. European Journal of Scientific Research. 2, (2009), 241-251.

[6] Zaid M. Odibat, Cyrille Aziz-Alaoui M. A., Bertelle \& H. E. Gérard. Duchamp. A multi-step differential transform method and application to non-chaotic or chaotic systems. Computers and Mathematics with Applications. 59, (2010), 1462-1472.

[7] S. A. Eftekhari \& A. A. Jafari. Numerical simulation of chaotic dynamical systems by the method of differential quadrature. Scientia Iranica B. 5, (2012), 1299-1315.

[8] Zhang Chengli \& Zeng Yun. A Simple Numerical Simulation Method for Lorenz System Families. Applied Mechanics and Materials. 444-445, (2014), 786-790.

[9] E. N. Lorenz. The predictability of hydrodynamic flow. Transactions of the New York Academy Sciences Series II. 25, (1963), 409-432.

[10] Eugenia Kalnay. Atmospheric Modeling, Data Assimilation and Predictability. New York: Cambridge University Press, 2002.

[11] Gilberto González-Parra, Luis Acedo \& Abraham J. Arenas. A novel approach to obtain analytical numerical solutions of nonlinear Lorenz system. Numerical Algorithms, 67, 2014, 93-107.

[12] Dimitri P. Bertsekas. Nedić, Angelia \& Ozdaglar, Asuman E. Convex analysis and optimization. Belmont: 
Athena Scientific, 2003.

[13] Morris W. Hirsch. Smale, Stephen \& Devaney, Robert L. Differential equations, dynamical systems, and an introduction to chaos (Third edition). Singapore: Elsevier (Singapore) Pte Ltd, 2015.

\section{Appendix}

Here, we give a proposition that when time step $\Delta \rightarrow 0$, the numerical solution in implicit time scheme are similar to explicit solution for a linear system.

Proof: For a linear system,

$$
F_{t}+F_{x}=0
$$

with the confirm boundary conditions, in explicit time scheme, the finite difference equation to this equation can be written as,

$$
\mathbf{F}_{j+1}=\mathbf{I F}_{j}+\Delta \mathbf{A} \mathbf{F}_{j},
$$

where subscript $j$ indicates $j \Delta, \mathbf{A}$ is invertible matrix, and $\mathbf{I}$ is identity matrix. In implicit time scheme, this equation can be written as,

$$
\mathbf{I F}_{j+1}-\Delta \mathbf{A F}_{j+1}=\mathbf{F}_{j},
$$

So, in implicit time scheme, the numerical solution can be written as,

$$
\mathbf{F}_{j+1}=(\mathbf{I}-\Delta \mathbf{A})^{-1} \mathbf{F}_{j},
$$

With the initial condition $\mathbf{F}_{0}$, both numerical solutions can be written as,

$$
\begin{aligned}
& \text { implicit solution: } \mathbf{F}_{n}=(\mathbf{I}-\Delta \mathbf{A})^{-n} \mathbf{F}_{0} \\
& \text { explicit solution: } \mathbf{F}_{n}=(\mathbf{I}+\Delta \mathbf{A})^{n} \mathbf{F}_{0}
\end{aligned}
$$

As $\mathbf{A}$ is invertible matrix, we can write this matrix as,

$$
\mathbf{A}=\mathbf{U} \mathbf{\wedge} \mathbf{V},
$$

where $\mathbf{U V}=\mathbf{I}, \boldsymbol{\Lambda}$ is diagonal matrix and as we known, $\boldsymbol{\Lambda}_{i j}=0$ as $i \neq j$. So, both solutions can be written as,

$$
\left\{\begin{array}{l}
\mathbf{F}_{n}=\mathbf{U}(\mathbf{I}-\Delta \mathbf{\Lambda})^{-n} \mathbf{V} \mathbf{F}_{0}, \\
\mathbf{F}_{n}=\mathbf{U}(\mathbf{I}+\Delta \mathbf{\Lambda})^{n} \mathbf{V F}_{0}
\end{array}\right.
$$

For the $\mathbf{I}-\Delta \mathbf{\Lambda}$, the invertible matrix is

$$
(\mathbf{I}-\Delta \mathbf{\Lambda})^{-1}=\frac{1}{\mathbf{I}-\Delta \mathbf{\Lambda}}=\mathbf{D},
$$


where $\mathbf{D}_{i i}=\left(1-\Delta \mathbf{\Lambda}_{i i}\right)^{-1}$, and $\mathbf{D}_{i j}=0$ as $i \neq j$. When the time step $\Delta \rightarrow 0$, this matrix can be written as,

$$
\mathbf{D}_{i i} \approx 1+\Delta \mathbf{\Lambda}_{i i}+O\left(\Delta^{2}\right)
$$

This matrix is $\mathbf{I}+\Delta \mathbf{\Lambda}$; the implicit solution can be approximately written as,

$$
\mathbf{F}_{n}=\mathbf{U}(\mathbf{I}+\Delta \mathbf{\Lambda})^{n} \mathbf{V F}_{0}+n O\left(\Delta^{2}\right) \mathbf{U}(\mathbf{I}+\Delta \mathbf{\Lambda})^{n-1} \mathbf{V F}_{0}+\ldots
$$

We can find that the first right term is the explicit solution, as time step $\Delta \rightarrow 0$, we can neglect the high order terms, such as $O\left(\Delta^{2}\right)$, so the implicit solution can be written as the explicit solution. 